\title{
Engaging in an auditory task suppresses responses in auditory cortex
}

\author{
Gonzalo H. Otazu ${ }^{1}$, Lung-Hao Tai ${ }^{1,2}$, Yang Yang ${ }^{1,2}$, and Anthony M. Zador ${ }^{1}$ \\ ${ }^{1}$ Cold Spring Harbor Laboratory, 1 Bungtown Road, Cold Spring Harbor, NY 11724 \\ ${ }^{2}$ Graduate Program in Neuroscience, Stony Brook University, NY 11794
}

\begin{abstract}
Although systems involved in attentional selection have been studied extensively, much less is known about non-selective systems. To study these preparatory mechanisms, we compared activity in auditory cortex elicited by sounds while rats performed an auditory task ("engaged") with activity elicited by identical stimuli while subjects were awake but not performing a task ("passive"). Surprisingly, we found that engagement suppressed responses, an effect opposite in sign to that elicited by selective attention. In the auditory thalamus, however, engagement enhanced spontaneous firing rates but did not affect evoked responses. These results demonstrate that in auditory cortex, neural activity cannot be viewed simply as a limited resource allocated in greater measure as the state of the animal passes from somnolent to passively listening to engaged and attentive. Instead the engaged condition possesses a characteristic and distinct neural signature in which sound-evoked responses are paradoxically suppressed.
\end{abstract}

Using sensory cues to drive purposeful activity requires sufficient levels of arousal and attention. The neural mechanisms of arousal have been studied by comparing neural activity in an awake state to activity recorded during sleep or anesthesia ${ }^{1-4}$. These studies have revealed that the neural signatures of unaroused brain states, including slow wave and REM sleep, are different from those of the awake condition.

Attention is itself complex, consisting of a well-defined selective component and a much less well-defined component encompassing arousal, vigilance and sustained attention. The neural mechanisms of selective attention are studied in paradigms in which a subject must base its behavior on one out of several sensory stimuli. These studies reveal that selective attention has a characteristic neural signature typically consisting of an increase in responsiveness to the attended stimulus ${ }^{5-10}$.

In contrast to the extensive body of literature on sleep and selective attention, little is known about the neural correlates of non-selective components of attention ${ }^{11}$. To study the neural

Users may view, print, copy, and download text and data-mine the content in such documents, for the purposes of academic research, subject always to the full Conditions of use:http://www.nature.com/authors/editorial_policies/license.html\#terms

Author contributions

G.H.O. and A.M.Z. designed the overall experiments and wrote the manuscript. G.H.O. designed and performed the experiments in Fig. 1, 2, 4 and 6. L.T. designed and performed the experiments in Fig. 3 (intermodal attention). Y.Y. designed and performed the experiments in Fig. 4 (head-fixed behavior). 
correlates of one of these non-selective components in auditory cortex, we compared cortical activity elicited by auditory stimuli in animals engaged in an auditory task with activity elicited by identical stimuli when an animal was passive but wakeful; we use the term "engaged" to refer to this non-selective component. Surprisingly, we found that engaging in an auditory task suppresses stimulus-evoked responses in the auditory cortex, in contrast to selective attention which, consistent with previous reports, enhances responses. We propose that suppression represents the wakeful baseline condition upon which other forms of attentional and non-attentional modulation are superimposed.

\section{Results}

In a first series of experiments, we compared responses elicited by acoustic stimuli in the auditory cortex when the animal was passive to those elicited when it was engaged. In a second series of experiments, we examined responses in the auditory cortex elicited by auditory stimuli during an intermodal (auditory-olfactory) selective attention task. In a third series of experiments, we compared responses during sleep and under anesthesia. In a fourth set of experiments, we compared cortical responses in the passive vs. the engaged condition in a modified version of the task in which the subject did not initiate trials. Finally, we compared passive and engaged responses in the auditory thalamus.

\section{Responses are suppressed in the engaged condition}

We trained adult male Long Evans rats to perform a two-alternative choice auditory discrimination task ${ }^{12,13}$. The animal initiated a trial by inserting its nose into the center port of a three port operant chamber (Fig. 1a and Methods: Task 1). After a waiting period ( 2 seconds), a "target" sound was presented from either the right or the left side of the box, indicating which goal port (right or left) would be rewarded with water. The target stimulus consisted of a $300 \mathrm{msec}$ broadband stimulus presented monoaurally from either the right or the left speaker. Later in the training phase, we introduced "non-target" stimuli during the waiting period, allowing us to probe the response to the non-target stimulus without retraining the animal on a new target. The non-target stimulus consisted of a train of clicks (5 msec white noise bursts; repetition rate from $2-35 \mathrm{~Hz}$, a range over which cortical responses show strong modulation ${ }^{14,15}$ ) presented diotically for 1.8 seconds, beginning after a variable (400-600 msec) period following trial initiation. The onset of the non-target stimulus was randomized with respect to the nose poke, so the animal could not predict the stimulus onset precisely. After animals reached criterion performance (> 95\% correct; 1 week), we implanted movable tetrodes in the left primary auditory cortex (area A1) to record neural activity, including single unit responses, multi-unit responses and local field potentials (LFPs). We also implanted earphones to ensure delivery of a controlled auditory stimulus to the unrestrained animal, regardless of its position in the box.

We first compared sound-evoked neural activity when the animal was engaged in the task with activity elicited by the same stimulus when the animal was passive but not asleep (see SM1: Behavioral monitoring and Fig. S1: Video analysis of movement). Because the sounds were delivered through headphones, differences in neural activity in this paradigm must be 
due to differences in the animal's behavior or state, rather than to differences in the stimulus itself arising from uncontrolled changes in the sound path from speaker to the ear.

We expected that engagement in an auditory task would, like selective attention, lead to an enhancement of stimulus-evoked responses in the auditory cortex ${ }^{8,16-19}$. Surprisingly, we found just the opposite: in the engaged condition, neural responses to all components of the stimulus — both target and non-target — were consistently suppressed.

We first analyzed the responses elicited by the first non-target stimulus (Fig. 1b-d). Fig. 1b shows suppression of the sound-evoked response in a well-isolated single unit. Suppression of the evoked response was observed for most of the responsive (SM2: Stimulus response analysis) single units (Fig. 1e, $\mathrm{p}<0.01$ ). Suppression was also observed for measures of neural population activity, including multiunit activity (Fig. 1f, $\mathrm{p}<1 \mathrm{e}-16$ ) and evoked LFP (Fig. 1g, p<1e-16). We quantified these effects across the population with a modulation index, defined as: (Activity engaged $_{-}$Activity $\left._{\text {passive }}\right) /\left(\right.$ Activity $_{\text {engaged }}+$ Activity $\left._{\text {passive }}\right)$. The modulation index ranges from -1 (complete suppression in the engaged condition) to +1 (complete suppression in the passive condition). The modulation index was significantly negative for all three physiological measures of the evoked response (Fig. 1h-j), indicating that engaging in an auditory task reduced the number of spikes used for stimulus representation. This suppression of evoked responses was not accompanied by any change in the spontaneous activity preceding stimulus presentation (Fig. $1 \mathrm{~b}-\mathrm{c}, \mathrm{e}-\mathrm{f}, \mathrm{h}-\mathrm{i}$ ), in contrast to other studies reporting an increase in spontaneous activity with task engagement ${ }^{19}$. There was no change ( $p>0.2)$ in neural activity between the first and second passive block of trials (i.e. between the passive block before and after the engaged block), indicating that suppression was not due to nonstationarities in the recording, satiety, reward expectation, arousal, etc. (see SM4: Comparison of neural activity between first and second passive block).

Although most single units showed a suppression of evoked activity in the engaged condition, a minority showed an increase (8/32 units). This had the effect of concentrating the stimulus-evoked spikes into smaller population of neurons, each with a relatively higher firing rate. This concentration of activity has been quantified in terms of the kurtosis, which is a measure of the sparseness of a neural representation. The kurtosis of the firing rate distribution in the engaged condition was greater than in the passive condition ( $_{\text {(urtosis }}$ engaged $=8.4 \pm 2.4$ vs. kurtosis passive $=4.1 \pm 0.7 ; \mathrm{p}<0.05$; see SM5: Kurtosis of firing rate distribution), indicating that the stimulus representation in the engaged condition was sparser.

We next analyzed the responses to the remaining non-target stimuli (Fig. 2a). As has been previously reported ${ }^{14,15}$, responses to the click train diminished as a function of repetition rate. However, at all but the highest repetition rates, the responses in the engaged condition were relatively suppressed relative to the passive condition (Fig. 2b). Thus suppression interacts with repetition rate, but is not limited to the initial stimulus.

Finally, we analyzed the response to the target stimulus. The train of non-target (diotic) stimuli preceding the target provided no information about the location of the reward, and 
was therefore irrelevant to the performance of the task; only the final (monaural) target stimulus was relevant and required the animal to attend to it. This raised the possibility that the previous results could be explained by a model in which the animal selectively withdraws its attention prior to the onset of the target stimulus. According to this hypothesis, suppression would be limited to the non-target stimuli, and the response to the relevant target stimulus would be enhanced. However, the response to the target stimulus was not enhanced (Fig. 2c; see also Fig. S2a-b: Single unit responses to task relevant and task irrelevant stimuli are equally suppressed during the task). Rather, the suppression of the responses to the target stimulus was indistinguishable (Fig. 2d-e and Fig. S2c: Single unit responses to task relevant and task irrelevant stimuli are equally suppressed during the task) from that of the last non-target stimulus in the stimulus train (paired t-test, $p>0.1$ ). The absence of response enhancement to the target stimulus is consistent with the modest attentional load in this task; had the attentional load been greater, the suppression might have been attenuated or even converted into enhancement (assuming that attentional mechanisms are distinct and additive). The equal suppression associated with target and non-target stimuli, and the lack of correlation between suppression and target selectivity, suggests that engagement induces a widespread suppression of evoked activity upon which any selective attentional effect is superimposed.

\section{Intermodal auditory attention does not suppress responses in auditory cortex}

Comparing the passive and engaged conditions revealed that sound-evoked responses were suppressed when the animal was engaged in an auditory task relative to those evoked when it was not performing the task. However, it was unclear from this comparison what the key difference between the two behavioral conditions was. One possibility was that, as suggested above, the key difference was whether the animal was engaged or passive; according to this hypothesis, suppression resulted simply from engaging in the task, regardless of the modality of the task. Alternatively, the suppression might result specifically from the engagement of the auditory cortex in this auditory task. Although this hypothesis would seem to contradict the intuitive expectation that engaging should increase rather than decrease neural activity, it is compatible with the data presented thus far, and makes a clear prediction: suppression in auditory cortex should not be observed if the animal were engaged but attending to a stimulus from a different sensory modality.

To distinguish these possibilities, we trained an additional group of five rats on an intermodal attention task ${ }^{20}$ in which we could compare sound-evoked responses elicited in auditory cortex when the animal was engaged in an auditory task with responses elicited by the same sounds when the animal was engaged in a non-auditory (olfactory) task (see Methods: Task 2). An auditory stimulus and an olfactory stimulus were presented simultaneously on every trial. To increase the difficulty of the task and thereby increase the likelihood of detecting an effect of selective attention ${ }^{21}$, we used a tone discrimination task which was substantially more challenging $(\sim 3$ weeks training, performance $\sim 80 \%)$ than the spatial discrimination task ( 1 week training, performance $>95 \%)$ presented above. Trials were grouped into olfactory and auditory blocks $(\sim 50-70$ trials each) in which the rat was rewarded for basing its decision on either the olfactory or the auditory stimulus, respectively. We reasoned that if the suppression observed in the engaged condition were 
specific to engaging in an auditory task, then responses in the auditory block would be suppressed relative to the olfactory block, whereas if suppression were not specific to the auditory nature of the task no difference would be observed between the two conditions. In these experiments, we defined the modulation index as: (Activity auditory block $^{-}$ Activity $\left._{\text {olfactory block }}\right) /\left(\right.$ Activity $_{\text {auditory block }}+$ Activity $\left._{\text {olfactory block }}\right)$.

There was no net suppression of sound-evoked responses elicited during the auditory block compared with those elicited during the olfactory block (Fig. 3a-b). Spontaneous activity was also not affected. Indeed, there was a slight, albeit nonsignificant, trend toward enhancement in the auditory block, as expected from recordings in an auditory area during an auditory task. A subset of single neurons showed strong modulation (either positive or negative) between the auditory and olfactory blocks, so that the very small net change between the blocks reflected a balance between the changes in the two conditions (not shown; see ${ }^{22}$ ). These results are inconsistent with the hypothesis that engaging in an auditory task per se suppresses responses in auditory cortex, and support the interpretation advanced above that suppression results from engaging in a task, regardless of sensory modality.

As a further test, we compared responses in all three conditions (passive, engaged-auditory, engaged-olfactory) in a single animal performing another intermodal attention task (see Methods: Task 3). As expected, sound-evoked responses were suppressed in both the auditory and the olfactory blocks compared with the passive block, and responses during the auditory block were not suppressed relative to the responses during the olfactory block (Fig. $3 c-d)$. Thus, engaging in an auditory task did not elicit a reduction in acoustically-evoked responses relative to engaging in an olfactory task, in marked contrast to the robust suppression of acoustically-evoked responses relative to the passive condition. We therefore conclude that the general suppression observed in the purely auditory task did not depend on the auditory nature of the task.

\section{Suppression is independent of receptive field properties}

In the visual cortex, attention can suppress responses in neurons that are not tuned to the attended feature ${ }^{23}$. Because in our experiments we recorded from several neurons simultaneously, we could not optimize the stimulus properties to match the receptive field properties of the recorded neurons. This raised the possibility that the suppression we observed in the engaged condition was due to attentional suppression in the majority of neurons which were stimulated suboptimally.

To test this hypothesis, we recorded tuning curves and assessed the relationship between the receptive field and the modulation index of the evoked responses. We expected that if suppression arose from the presentation of suboptimal stimuli, then the modulation index should increase with stimulus selectivity or with the degree to which the best frequency matched the attended frequency. However, there was no correlation between the modulation index and spatial selectivity (Fig. 2f and Fig. S2d: Single unit responses to task relevant and task irrelevant stimuli are equally suppressed during the task), frequency selectivity (Fig. $3 \mathrm{e}$ ), or the distance in octaves between the best frequency and the attended frequency (Fig. $3 \mathrm{f}$ and Fig. S3: Measurement of cortical tuning curves). Interestingly, suppression was 
correlated with spontaneous activity, a property of the cells that does not depend on the stimulus used during the task (see Fig. S4: Cortical units with high spontaneous firing rates show less suppression of the evoked response during the task). Thus in summary, taskengaged suppression cannot be readily predicted from receptive field properties.

\section{Changes in arousal have a distinct neural signature}

Gross changes in arousal, such as those arising from sleep or anesthesia, can cause major changes in neural responsiveness ${ }^{2,24}$. Although we were careful to exclude from the passive condition periods when the animal was immobile for an extended period and therefore possibly drowsy or asleep, the passive condition might nevertheless have been associated with a general decrease in arousal. However, this did not seem likely to explain the suppression we observed, since the increase in arousal in the engaged condition would have been expected to increase cortical excitability and thereby increase evoked or spontaneous firing rates ${ }^{2,3,19}$, effects opposite in sign from those we observed.

To characterize further the differences between the passive condition and previously described changes associated with arousal, in some sessions we compared neural activity in the passive condition to activity recorded either during prolonged periods of immobility which may have included sleep episodes (Fig 4a-c) or during ketamine anesthesia (Fig 4df). Spontaneous and evoked cortical activity showed a characteristic neural signature under each condition (Table T1): spontaneous cortical activity was reduced in the less aroused states (sleep and anesthetized) compared with the awake but passive condition. The distinct neural signatures associated with sleep and anesthesia indicate that these states are different from the passive but wakeful condition.

\section{Suppression of evoked responses is not caused by self-triggering of stimulus}

Previous studies in auditory cortex have shown that self-triggering of auditory stimulus, for example during vocalizations ${ }^{25,26}$, produces a reduction in the evoked responses, compared to when the subject hears the same stimulus without triggering it ${ }^{27}$. In all the behavioral paradigms that we have tested so far, during the engaged condition the animal triggered the stimulus by inserting its nose in the center port, as opposed to the passive condition, in which the stimulus was not triggered by the animal. To test whether self-triggering produced the suppression that we observed in the engaged condition, we trained 4 rats (Fig. 5a and Methods: Task 4) to perform a Go/No-Go task in the head-fixed configuration; in this version of the task, the animal did not trigger the stimulus in the engaged condition, but instead the stimulus started randomly. During the passive condition, the water delivery system was withdrawn and the animal heard the same stimulus as in the engaged condition. We found that evoked responses, both multiunit (Fig. 5b-c-d) and LFP (Fig. S5: Example and population data showing suppression of sound evoked LFP in the head-fixed behavior), were suppressed during the task, without changes in the spontaneous activity. The modulation index of the evoked responses for multiunit activity was $-0.20 \pm 0.03$, which was very similar to the values obtained in the task in Fig. 1, $-0.19 \pm 0.02$. We therefore conclude that the suppression observed in the engaged condition does not depend on the animal triggering the stimulus. 


\section{Engagement enhances spontaneous activity in auditory thalamus}

What circuit-level mechanisms might be responsible for the suppression seen during engagement? The auditory cortex is modulated by a rich system of neuromodulators and receives input from both lemniscal and non-lemniscal thalamic pathways ${ }^{28}$. To test whether task-engaged suppression was inherited from earlier levels in the auditory hierarchy, we recorded responses in the auditory thalamus (medial geniculate gody, see Fig. S6: Histological reconstruction of recordings in the medial geniculate body (MGB)) of two additional animals performing the task described in Fig. 1. In contrast to cortex, there was no difference $(\mathrm{p}=0.80)$ in the thalamic evoked response (Fig. 6a) between the engaged and passive conditions. However, the thalamic spontaneous activity showed a robust and consistent elevation in the engaged condition (Fig. 6b-c; p <0.001). No changes were observed in the burstiness of the thalamic cells (SM7: Bursts in thalamus), in contrast to elevated burstiness associated with sleep or reduced arousal ${ }^{29-32}$. This increase in spontaneous activity is consistent with the increase in the cortical LFP power (Fig. S7: Power spectrum of the spontaneous cortical activity in the passive and the engaged condition), since the LFP is often assumed to reflect synaptic activity in an area ${ }^{33,34}$.

Synaptic depression of the thalamocortical inputs 15,35 could explain how the relative enhancement of the thalamic spontaneous rate in the engaged condition might lead to suppression of the cortical evoked response, without changing the cortical spontaneous activity. Synaptic depression has previously been implicated in a range of functions in sensory processing, including gain control ${ }^{36}$. Thalamocortical synapses show synaptic depression at high firing rates 15,35 . At sufficiently high firing rates, synaptic release probability is inversely proportional to the presynaptic firing rate ${ }^{36}\left(\mathrm{P}_{\text {release }} \propto 1 / \mathrm{F}_{\text {thalamic }}\right)$. Under these conditions, the increase in the steady-state thalamic spontaneous firing rate in the engaged condition would not lead to a corresponding increase in the cortical spontaneous rate ${ }^{24}$, as the increased thalamic firing rate would be compensated by a decreased synaptic release probability. Furthermore, evoked responses of identical magnitude in thalamus would lead to relatively depressed responses in the cortex in the engaged condition, because the thalamocortical synapses would be in a more depleted state due to the preceding spontaneous activity (for details, see SM8: Thalamocortical depression model). Synaptic depression can also account for the reduction in the steady-state modulation index at high stimulation rates (Fig. 2a-b). Synaptic depression has also been implicated in the barrel cortex, where suppression has been observed during whisking ("engaged") compared with quiet wakefulness ("passive"), consistent with the present findings ${ }^{37-39}$; this effect can be mimicked in anesthetized rats by stimulation of the reticular formation or by application of acetylcholine in cortex ${ }^{40,41}$ and thalamus ${ }^{42}$ (see also Fig. 4d-f). Thus synaptic depression may account for the steady-state changes in the response to repetitive stimuli, but other mechanisms like feedforward inhibition or axon excitability ${ }^{43}$ may play a role during the first few stimuli at high repetition rates ${ }^{15}$.

\section{Discussion}

We have compared sound-evoked responses elicited during an auditory task with those elicited when the animal was wakeful but passive. We found that the neural signature of task 
engagement in the auditory cortex was a widespread and robust suppression of the evoked responses for both target and non-target stimuli, without any concomitant change in spontaneous activity. Experiments using an intermodal auditory-olfactory task, and in sleeping and anesthetized animals, showed that this suppression was specific to engaging in a task and could not be simply explained by different levels of arousal. Finally, we found that spontaneous activity in the auditory thalamus was enhanced during engagement, but evoked responses were unchanged. The thalamic recordings suggest that the mechanism for cortical suppression may involve depression at thalamocortical synapses.

Our central finding — that engaging in an auditory task suppresses rather than enhances activity in the auditory cortex - was initially surprising to us for two reasons. First, we expected that if the transition from the wakeful and passive condition to the engaged condition were associated with an increase in arousal, then cortical firing rates would be higher in the engaged condition ${ }^{1,2}$. However, evoked firing rates were lower in the engaged condition, and spontaneous rates were unchanged. Indeed, diminished cortical activity consistent with decreased arousal was observed only after prolonged periods of immobility (Fig 4). Furthermore, in the passive condition there was no increase in thalamic bursting, as has been reported with reduced arousal ${ }^{29-32}$. Second, we expected that the difference between the engaged and passive conditions might recruit attentional mechanisms and thereby increase evoked responses (see Fig. 4 and ${ }^{5-9}$ ). Our results indicate that the passive but wakeful condition cannot be viewed simply as a point in a continuum of arousal states from sleep to active to attentive, along which neural excitability increases monotonically; the passive condition possesses instead a characteristic and distinct neural signature. Characterizing this signature represents a first step toward understanding the non-specific components of attention ${ }^{11}$.

Could the suppression associated with engagement be explained by previously described selective attention mechanisms acting on the engaged condition? Selective attention is usually reported to enhance neural responses ${ }^{5-10}$, but can also lead to suppression of nonoptimal stimuli ${ }^{23,44}$. Because we recorded simultaneously with several tetrodes, we did not explicitly optimize the stimulus for any particular neuron, so most of the neurons from which we recorded were driven sub-optimally. However, selectively attending to suboptimal stimuli is not likely to account for the widespread suppression we describe. First, in the intermodal selective attention task (Fig. 3), in which target stimuli were similarly suboptimal, no widespread suppression was observed when the auditory stimulus was selectively attended. Intermodal attention enhanced responses in some auditory cortical neurons and suppressed it in others ${ }^{220}$, but it did not generate the large population effects observed in task-engaged suppression. Second, suppression resulting from engagement was general, for both target and non-target stimulus, and appeared independent of neural selectivity; responses selective for the location of the sound were not preferentially enhanced during the sound localization task (Fig. $2 \mathrm{f}$ and Fig. S2d: Single unit responses to task relevant and task irrelevant stimuli are equally suppressed during the task), nor were responses selective for the sound frequency enhanced during the frequency discrimination task (see Fig. 3e-f). Third, effects of selective attention are often limited to the later components of the response ${ }^{45}$, whereas the suppression associated with engagement was evident even in the early onset response (see Fig. 1 and Fig. S8: Effects on modulation index 
in response to clicks were robust to perturbations in window size). Task-dependent suppression thus appears distinct from but compatible with the enhancement resulting from selective attention ${ }^{6,8}$, reward ${ }^{46}$, or other stimulus-specific processes.

Suppression in the rat barrel cortex is observed during whisking ("engaged") compared with quiet wakefulness ("passive"), consistent with the present findings ${ }^{37-39}$. (Interestingly, suppression of visual cortical responses during comparable passive viewing has not been widely reported, perhaps because the requisite visual fixation may represent a form of engagement.) This suppression results from the transition from a synchronized to a desynchronized cortical state, and can be mimicked by stimulation of the reticular formation or by cortical 24,41 or thalamic ${ }^{42}$ application of acetylcholine. However, differences in the behavioral paradigms used in the present work (sensory discrimination tasks modeled after primate studies) and the paradigms used in the barrel system studies (based on active exploration and passive stimulation) preclude a more detailed comparison between these results.

What might be the function of task-engaged suppression? When an animal is engaged in an auditory task, a sensory signal originating in the cochlea must ultimately be routed to motor centers to trigger the appropriate action, whereas in the passive condition the identical signal does not trigger the behavior. Neurons in the rat primary auditory cortex project to a wide range of targets, including the visual cortex, the posterior parietal cortex and the amygdala. Task-engaged suppression may represent an initial stage of this routing, in which activity in neurons irrelevant to the task is reduced. In the passive condition, in which no task has been defined, there is no well-defined population of neurons needed for the task, so it may be that the auditory signal is propagated to a wider range of target brain regions. This task-engaged modulation thus appears to be distinct from that observed in selective attention tasks, in which activity in a subset of neurons representing one stimulus is often boosted relative to that in neurons representing a competing stimulus; in our task the sensory component is far from threshold, so little boosting is needed and suppression emerges as the dominant mechanism. Our results represent a first step toward understanding the synaptic and circuit mechanisms by which this suppression occurs.

\section{Methods}

All experiments were conducted in a single-walled sound booth (Industrial Acoustics Company, Bronx, New York, USA). Animals were water deprived under a protocol approved by the Cold Spring Harbor Laboratory Animal Committee (see Supplementary table 1: Experiment summary). Subjects in all experiments were adult male Long Evans rats (Taconic Farms, Germantown NY, USA), with the exception of task 4 in which 30-35 day old animals were used.

Task 1 (Passive vs. engaged-auditory; Fig. 1,2 and 6)—In the engaged (task) condition, the subject was trained to poke its nose into the center port, thereby triggering the onset of the non-target stimulus, which consisted of a train of diotic clicks (white noise bursts, $5 \mathrm{~ms}$ duration), followed by the target stimulus. The onset of the train was preceded by a random delay of 400-600 msec. The non-target stimulus lasted for 1.8 seconds, after 
which the target stimulus was presented. The target stimulus consisted of a monoaural, $0.3 \mathrm{~s}$ broadband sound, formed by 16 tones between 1 and $16 \mathrm{kHz}$, uniformly distributed in the logarithmic space according to the formula $\mathrm{f}_{\mathrm{n}}=1000 *(1.203 \ldots)^{\mathrm{n}} \mathrm{Hz}$ for $\mathrm{n}=0,1 \ldots 15$. The subject remained in the center port until the end of target delivery. The target stimulus indicated the location of the reward port on that trial. Subjects performed a trial every $9.03 \pm 0.16 \mathrm{sec}$ (mean \pm s.e.m) for $\sim 200$ trials per recording session. In the passive condition, the three ports were blocked, and the same sequence of stimuli was delivered (every $9.37 \pm 0.28 \mathrm{sec}$; mean \pm s.e.m; $~ 100$ stimulus repetitions before and $\sim 100$ stimulus repetitions after the animal performed the task).

Task 2 (Engaged-auditory vs. engaged-olfactory; Fig. 3a-b)—Subjects were first trained to perform an auditory task. The task consisted of the discrimination between two pure tones, delivered free-field at 60-65 dB SPL for at least $50 \mathrm{msec}$. The low tone was chosen in the range of 5-7 kHz; the high tone was chosen in the range of 13-20 kHz. After a subject reached a performance of $>90 \%$, it was trained to perform an olfactory discrimination task that consisted of either the discrimination between caproic acid/hexanol (2 animals), $\mathrm{R}(-)-2$-octanol/S(+)-2-octanol (1 animal), or R(-)-2-octanol/S(+)-2-octanol mixture (ratio of 70/30 vs. 30/70, 2 animals). Training of auditory (A) and olfactory (O) discrimination tasks followed steps similar to those described in ${ }^{12}$.

We first trained subjects to perform both auditory and olfactory tasks in alternating blocks (AAA.../OOO...). We then trained rats to perform in alternating auditory-only blocks and olfactory blocks with sound distracters (AA.../OaOa.../AA...), which we call the halfsymmetrical task. We then introduced a null odor (caproic acid) in the auditory block and the animals performed a full symmetrical task (AoAo.../OaOa.../AoAo...). Two animals performed the full symmetrical task and three animals performed the half-symmetrical task. Results were similar and were pooled together. The blocks lasted for $\sim 50-70$ trials. Performance was $81 \%$ during the auditory blocks and $91 \%$ during the olfactory blocks, indicating that the subjects understood the block structures of the task.

Task 3 (Engaged-auditory vs. engaged-olfactory vs. passive; Fig. 3c-f)-We trained subjects to perform a modified version of the intermodal attention task (Task 2). This task used a 5-port operant chamber which consisted of upper and lower reward ports on both the right and left sides, in addition to the center port. The subject initiated the trial by inserting its nose in the center port, which triggered the stimulus after a variable (150-200 ms) delay. In the auditory block, the auditory stimulus consisted of two components presented sequentially. The first component (a diotic $65 \mathrm{~dB}$ SPL $400 \mathrm{~ms}$ pure tone, either $5612 \mathrm{~Hz}$ or $15874 \mathrm{~Hz}$ ) signaled whether the reward would be available at one of the upper or one of the lower ports. The second component (a monaural broadband sound from either the left or the right earphone presented 560-610ms after the first) signaled whether the reward would be available from the left or right port. In the olfactory block, the olfactory stimulus also consisted of two components presented sequentially, with (+)-fenchone vs. (-)fenchone signaling the upper or lower ports, respectively, and R(-)-2-octanol vs. S(+)-2octanol signaling the left or right ports, respectively. In the auditory block, only the auditory stimuli were presented, but in the olfactory block, the first component of the auditory 
stimulus (i.e. the $400 \mathrm{msec}$ pure tone) was also presented as a distractor. This design allowed us to compare neural responses to the first auditory component under conditions when attention was directed toward (auditory block) or away from (olfactory block) the auditory stimulus. In the passive condition, the same auditory stimuli were presented before the animal started doing the task ( 100 stimulus repetitions). In this period, we also measured the frequency tuning (see Fig. S3: Measurement of cortical tuning curves).

Task 4 (Passive vs. engaged-auditory, Fig. 5) -In the engaged-auditory condition, head-fixed subjects performed an auditory Go/No-Go task in which they were required to lick a water spout after presentation of a target sound and to refrain from licking after presentation of a distractor sound. Correct licks were rewarded with water, incorrect licks were discouraged with a mild air puff and a short (1-3 second) time out (see ${ }^{47}$ for details on training and other task details). The stimulus consisted of a train of 10 clicks $(20 \mathrm{~Hz}, 5 \mathrm{~ms}$ duration, $58 \mathrm{~dB}$ SPL RMS, $73 \mathrm{~dB}$ SPL peak value) followed either by a non-target (pure tone, $100 \mathrm{msec}, 24000 \mathrm{~Hz}$ ) or a target (amplitude-modulated warble, $6000 \mathrm{~Hz}$ carrier, $78 \mathrm{~dB}$ SPL RMS, 86 dB SPL peak) stimulus. The intertrial interval was formed by adding one second to a time chosen randomly from an exponential distribution (1 second decay). In the passive condition, the same stimuli with the same intertrial interval were presented, but the water spout was removed so subjects could not perform the task. The animals performed two blocks of 20 minutes (engaged periods), with an intermediate period, in which the water delivery system was withdrawn, but the same sounds were played (passive period).

\section{Surgery}

All procedures were approved by the Cold Spring Harbor Laboratory Animal Committee. Animals were anesthetized with an intraperitoneal injection of a mixture of ketamine (60 $\mathrm{mg} / \mathrm{kg})$ and medetomidine $(0.51 \mathrm{mg} / \mathrm{kg})$. Wounds were infiltrated with lidocaine. For tetrode implants in left auditory cortex (12 animals total, Fig. 1-4), the temporal muscle was recessed and a craniotomy and a durotomy were performed. Electrodes were implanted between 4.5 and $5.0 \mathrm{~mm}$ posterior to bregma and $6.4 \mathrm{~mm}$ left of the midline. For implants in left auditory thalamus ( 2 animals, Fig. 6), the coordinates were between 5.5 and $6.0 \mathrm{~mm}$ posterior from bregma and 3.2 to $4 \mathrm{~mm}$ left of the midline. The electrodes were advanced to a depth of $4 \mathrm{~mm}$ from the brain surface. We also attached a plastic ring next to but not touching each pinna, which we could use to screw the earphones into place. After surgery, animals were left to recover for several days before resuming water deprivation. The surgery for the head-fixed behavior has been previously described ${ }^{48}$. Briefly, 4 young rats ( $60 \mathrm{gr}$ ) were implanted with a well over the left auditory cortex and a post for head fixation.

\section{Electrophysiology}

For the freely moving animals, we implanted polyimide-coated nichrome wires (H.P. Reid, Inc., Palm Coast, Florida, USA; wire diameter $12.5 \mu \mathrm{m}$ ) twisted in groups of four as tetrodes (each wire gold plated to $<0.5 \mathrm{M} \Omega$ impedance at $1 \mathrm{kHz}$ ). We implanted six independently movable tetrodes using a custom-built drive. We recorded spiking activity and local field potentials (LFPs) with a Cheetah32 32 Channel System (Neuralynx, Inc, 34 North Pantano Road Tucson, Arizona, USA). For the head-fixed animals, we used a single tungsten electrode (Model TM33C10, World Precision Instruments, Sarasota, Florida, USA) with an 
impedance of $1 \mathrm{M} \Omega$, amplified using a CyberAmp 380 (Molecular Devices, Sunnyvale, California, USA) and recorded using Matlab custom software.

To detect spiking activity, we filtered the signal between $900 \mathrm{~Hz}$ and $6 \mathrm{kHz}$. When a threshold crossing event in any of the four leads was detected, a $1 \mathrm{~ms}$ waveform was acquired at $32 \mathrm{kHz}$. The sampled waveforms were automatically clustered using KlustaKwik (K. D. Harris et al.; http://klustakwik.sourceforge.net), using Peak6to11 (minimum of the value in samples 6 to samples 11), Valley (maximum of the voltage deflection) and Energy (L-2 norm of the acquired waveform) as clustering features. The clusters were later checked and adjusted manually using MClust (A. D. Redish et al.; http://mclust.sourceforge.net). Clusters were included in the analysis only if the following criteria were met: (1) <1\% refractory period violations; (2) an isolation distance ${ }^{49}$ (ID) of more than 15 , calculated based on Peak, Energy, FFT and PC1 (Principal Component 1); (3) clusters were stable for at least 100 trials during the engaged condition and at least 100 trials of passive conditions. The ID is defined as the Mahalanobis distance from the center of an identified cluster within which as many spikes belong to the specified cluster as others.

For the multiunit analysis, events were included if they exceeded a threshold of $50 \mu \mathrm{V}$ on any of the four channels of the tetrode. Re-analysis of the data presented in this paper by changing these thresholds between $35 \mu \mathrm{V}$ and $75 \mu \mathrm{V}$ did not change the results. Multiunit sites were included in the analysis only if there were at least 5 total spikes in the spontaneous period ( $20 \mathrm{~ms}$ before stimulus onset) and in the evoked period (20 ms after stimulus onset).

To obtain local field potentials (LFPs), we filtered the signal from one of the leads of each tetrode, or the tungsten electrode used for the head-fixed behavior between $1 \mathrm{~Hz}$ and 475 Hz. After acquisition at $3225 \mathrm{~Hz}$, we applied a high-pass 4-pole Butterworth filter $(10 \mathrm{~Hz})$.

Each day, each tetrode or tungsten electrode was independently advanced until we could observe stable spiking activity. We did not specifically sample for sites that were responsive to our stimulus ensemble. We advanced the tetrodes at least $40 \mu \mathrm{m}$ every day to avoid having multiple recording sessions with the same subset of cells. We used a skull screw as a ground. We used a nearby nichrome wire as a reference for the tetrode recordings, and another skull screw for the head-fixed recordings.

\section{Stimulus delivery}

For the stimulus delivery through earphones (Fig. 1, 2, 3c-f, 4 and 6) on each recording day, an earphone (ER*6i Isolator, Etymotic Research, Illinois, USA) was screwed into the earphone holder without anesthetizing the animal. The earphone had a soft silicone cover, which allowed us to adjust it in place without causing discomfort to the animal. Sound intensity was determined with a Brüel \& Kjær type 4939 free-field microphone, Type 2670 1/4-inch Microphone Preamplifier and Type 2690A0S2 2-Channel Microphone Conditioning Amplifier (Brüel \& Kjær Sound \& Vibration Measurement A/S, Denmark) positioned $5 \mathrm{~mm}$ in front of the earphone. At this position, the intensity of the chord was 69dB RMS SPL (74 dB SPL peak value), and the click was 76dB RMS SPL ( $82 \mathrm{~dB}$ SPL peak value). 
For animals recorded during free-field stimulation (Fig. 3a-b), the stimulus was played through a calibrated PC speaker located $6 \mathrm{~cm}$ in front of the animal's head. For the headfixed behavior (Fig. 5), we used an electrostatic speaker (ES1, Tucker-Davis Technologies, Florida, USA) placed on the right side, $10 \mathrm{~cm}$ from the ear.

\section{Supplementary Material}

Refer to Web version on PubMed Central for supplementary material.

\section{Acknowledgments}

This work was supported by grants from the National Institutes of Health, the Swartz Foundation, the Marie Robertson Fund, the Louis Morin Charitable Trust, and the Coleman Fung Foundation.

\section{References}

1. Steriade M, Timofeev I, Grenier F. Natural waking and sleep states: a view from inside neocortical neurons. J Neurophysiol. 2001; 85:1969-85. [PubMed: 11353014]

2. Edeline JM, Dutrieux G, Manunta Y, Hennevin E. Diversity of receptive field changes in auditory cortex during natural sleep. Eur J Neurosci. 2001; 14:1865-80. [PubMed: 11860482]

3. Talwar SK, Gerstein GL. Reorganization in Awake Rat Auditory Cortex by Local Microstimulation and Its Effect on Frequency-Discrimination Behavior. J Neurophysiol. 2001; 86:1555-1572. [PubMed: 11600620]

4. Castro-Alamancos MA. Dynamics of sensory thalamocortical synaptic networks during information processing states. Prog Neurobiol. 2004; 74:213-47. [PubMed: 15556288]

5. Moran J, Desimone R. Selective attention gates visual processing in the extrastriate cortex. Science. 1985; 229:782-4. [PubMed: 4023713]

6. Desimone R, Duncan J. Neural mechanisms of selective visual attention. Annu Rev Neurosci. 1995; 18:193-222. [PubMed: 7605061]

7. Maunsell JH, Cook EP. The role of attention in visual processing. Philos Trans R Soc Lond B Biol Sci. 2002; 357:1063-72. [PubMed: 12217174]

8. Fritz J, Shamma S, Elhilali M, Klein D. Rapid task-related plasticity of spectrotemporal receptive fields in primary auditory cortex. Nat Neurosci. 2003; 6:1216-23. [PubMed: 14583754]

9. Fritz JB, Elhilali M, David SV, Shamma SA. Does attention play a role in dynamic receptive field adaptation to changing acoustic salience in A1? Hear Res. 2007; 229:186-203. [PubMed: 17329048]

10. Ghose GM, Maunsell JH. Attentional modulation in visual cortex depends on task timing. Nature. 2002; 419:616-20. [PubMed: 12374979]

11. Raz A, Buhle J. Typologies of attentional networks. Nat Neurosci Reviews. 2006; 7:367-379.

12. Uchida N, Mainen ZF. Speed and accuracy of olfactory discrimination in the rat. Nat Neurosci. 2003; 6:1224-9. [PubMed: 14566341]

13. Yang Y, DeWeese MR, Otazu GH, Zador AM. Millisecond-scale differences in neural activity in auditory cortex can drive decisions. Nat Neurosci. 2008; 11:1262-3. [PubMed: 18849984]

14. Kilgard MP, Merzenich MM. Plasticity of temporal information processing in the primary auditory cortex. Nat Neurosci. 1998; 1:727-31. [PubMed: 10196590]

15. Wehr M, Zador AM. Synaptic mechanisms of forward suppression in rat auditory cortex. Neuron. 2005; 47:437-45. [PubMed: 16055066]

16. Hubel DH, Henson CO, Rupert A, Galambos R. "Attention” Units in the Auditory Cortex. Science. 1959; 129:1279-1280. [PubMed: 13658956]

17. Picton TW, Hillyard SA, Galambos R, Schiff M. Human Auditory Attention: A Central or Peripheral Process? Science Vol. 1971; 173:351-353. 
18. Fritz JB, Elhilali M, Shamma SA. Adaptive changes in cortical receptive fields induced by attention to complex sounds. J Neurophysiol. 2007; 98:2337-46. [PubMed: 17699691]

19. Scott BH, Malone BJ, Semple MN. Effect of behavioral context on representation of a spatial cue in core auditory cortex of awake macaques. J Neurosci. 2007; 27:6489-99. [PubMed: 17567810]

20. Hocherman S, Benson DA, Goldstein MH Jr, Heffner HE, Hienz RD. Evoked unit activity in auditory cortex of monkeys performing a selective attention task. Brain Res. 1976; 117:51-68. [PubMed: 825193]

21. Spitzer H, Desimone R, Moran J. Increased attention enhances both behavioral and neuronal performance. Science. 1988; 240:338-40. [PubMed: 3353728]

22. Tai, LH.; Zador, A. Neural Mechanisms of Selective Auditory Attention in Rats (Dissertation). Available from Nature Precedings. 2008. <http://dx.doi.org/10.1038/npre.2008.2355.1>

23. Treue S, Martinez Trujillo JC. Feature-based attention influences motion processing gain in macaque visual cortex. Nature. 1999; 399:575-9. [PubMed: 10376597]

24. Castro-Alamancos MA, Oldford E. Cortical sensory suppression during arousal is due to the activity-dependent depression of thalamocortical synapses. J Physiol. 2002; 541:319-31. [PubMed: 12015438]

25. Muller-Preuss P, Ploog D. Inhibition of auditory cortical neurons during phonation. Brain Res. 1981; 215:61-76. [PubMed: 7260601]

26. Eliades SJ, Wang X. Neural substrates of vocalization feedback monitoring in primate auditory cortex. Nature. 2008; 453:1102-6. [PubMed: 18454135]

27. Martikainen MH, Kaneko K, Hari R. Suppressed responses to self-triggered sounds in the human auditory cortex. Cereb Cortex. 2005; 15:299-302. [PubMed: 15238430]

28. Boly M, et al. Baseline brain activity fluctuations predict somatosensory perception in humans. Proc Natl Acad Sci U S A. 2007; 104:12187-92. [PubMed: 17616583]

29. Reinagel P, Godwin D, Sherman SM, Koch C. Encoding of visual information by LGN bursts. J Neurophysiol. 1999; 81:2558-69. [PubMed: 10322089]

30. Sherman SM. Thalamic relays and cortical functioning. Prog Brain Res. 2005; 149:107-26. [PubMed: 16226580]

31. Swadlow HA, Gusev AG. The impact of 'bursting' thalamic impulses at a neocortical synapse. Nat Neurosci. 2001; 4:402-8. [PubMed: 11276231]

32. Massaux A, Dutrieux G, Cotillon-Williams N, Manunta Y, Edeline JM. Auditory thalamus bursts in anesthetized and non-anesthetized states: contribution to functional properties. J Neurophysiol. 2004; 91:2117-34. [PubMed: 14724263]

33. Poulet JF, Petersen CC. Internal brain state regulates membrane potential synchrony in barrel cortex of behaving mice. Nature. 2008; 454:881-5. [PubMed: 18633351]

34. Deweese MR, Zador AM. Shared and private variability in the auditory cortex. J Neurophysiol. 2004; 92:1840-55. [PubMed: 15115790]

35. Chung S, Li X, Nelson S. Short-Term Depression at Thalamocortical Synapses Contributes to Rapid Adaptation of Cortical Sensory Responses In Vivo. Neuron. 2002; 34:437-446. [PubMed: 11988174]

36. Abbott LF, Varela JA, Sen K, Nelson SB. Synaptic depression and cortical gain control. Science. 1997; 275:220-4. [PubMed: 8985017]

37. Crochet S, Petersen CC. Correlating whisker behavior with membrane potential in barrel cortex of awake mice. Nat Neurosci. 2006; 9:608-10. [PubMed: 16617340]

38. Ferezou I, Bolea S, Petersen CC. Visualizing the cortical representation of whisker touch: voltagesensitive dye imaging in freely moving mice. Neuron. 2006; 50:617-29. [PubMed: 16701211]

39. Fanselow EE, Nicolelis MA. Behavioral modulation of tactile responses in the rat somatosensory system. J Neurosci. 1999; 19:7603-16. [PubMed: 10460266]

40. Castro-Alamancos MA. Role of thalamocortical sensory suppression during arousal: focusing sensory inputs in neocortex. J Neurosci. 2002; 22:9651-5. [PubMed: 12427819]

41. Castro-Alamancos MA. Absence of rapid sensory adaptation in neocortex during information processing states. Neuron. 2004; 41:455-64. [PubMed: 14766183] 
42. Hirata A, Castro-Alamancos MA. Relief of synaptic depression produces long-term enhancement in thalamocortical networks. J Neurophysiol. 2006; 95:2479-91. [PubMed: 16381803]

43. Kawai H, Lazar R, Metherate R. Nicotinic control of axon excitability regulates thalamocortical transmission. Nat Neurosci. 2007; 10:1168-75. [PubMed: 17704774]

44. Reynolds JH, Chelazzi L, Desimone R. Competitive mechanisms subserve attention in macaque areas V2 and V4. J Neurosci. 1999; 19:1736-53. [PubMed: 10024360]

45. Cook EP, Maunsell JH. Dynamics of neuronal responses in macaque MT and VIP during motion detection. Nat Neurosci. 2002; 5:985-94. [PubMed: 12244324]

46. Shuler MG, Bear MF. Reward timing in the primary visual cortex. Science. 2006; 311:1606-9. [PubMed: 16543459]

47. Hromadka, T. Representation of Sounds in Auditory Cortex of Awake Rats (Dissertation). Available from Nature Precedings. 2008. <http://dx.doi.org/10.1038/npre.2008.2464.1>

48. Hromadka T, Deweese MR, Zador AM. Sparse representation of sounds in the unanesthetized auditory cortex. PLoS Biol. 2008; 6:e16. [PubMed: 18232737]

49. Schmitzer-Torbert N, Jackson J, Henze D, Harris K, Redish AD. Quantitative measures of cluster quality for use in extracellular recordings. Neuroscience. 2005; 131:1-11. [PubMed: 15680687]

50. Feierstein CE, Quirk MC, Uchida N, Sosulski DL, Mainen ZF. Representation of spatial goals in rat orbitofrontal cortex. Neuron. 2006; 51:495-507. [PubMed: 16908414] 


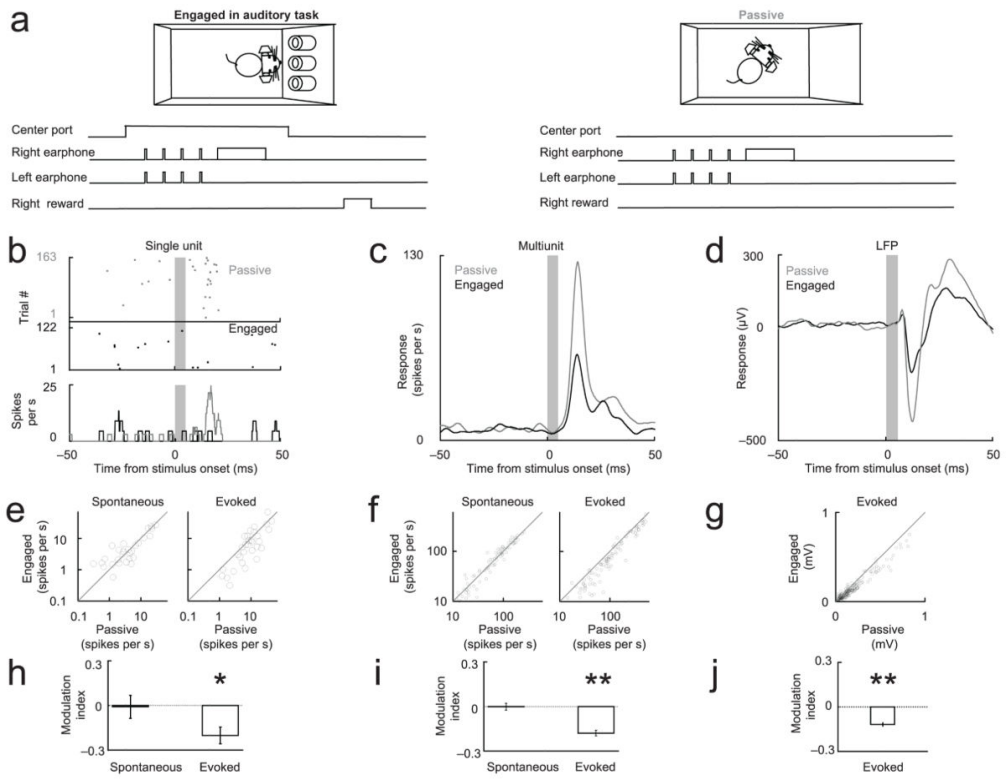

Figure 1. Cortical evoked responses are suppressed in the engaged condition but spontaneous activity is unchanged

(a) Animals implanted with earphones performed a two-alternative choice auditory task (Task 1) for $\sim 30$ minutes (engaged period). The animal initiated a trial by poking its nose into the center port. Before and after the engaged period, the ports were blocked and the same stimuli were presented (passive period). (b-d) Examples of single unit, multi-unit and LFP responses elicited by the first stimulus ( grey bar) showing suppression in engaged relative to passive condition. Fig. $\mathbf{e}-\mathbf{h}, \mathbf{f}-\mathbf{i}$ and $\mathbf{g}-\mathbf{j}$ show population responses of single units, multiunits and LFP respectively. (e-g) Scatterplot comparing passive and engaged activity across the population. (h-j) Modulation index ((Activity engaged $^{-}$Activity $\left._{\text {passive }}\right) /$ $\left(\right.$ Activity $_{\text {engaged }}+$ Activity $\left._{\text {passive }}\right)$ ) for spontaneous and evoked activity. Because LFP changes were assessed by changes in the stimulus-evoked peak, LFP spontaneous activity was not analyzed in $\mathbf{g}$ and $\mathbf{j}$. ( $*-\mathrm{p}<0.05$ different from $0 ; * *-\mathrm{p}<0.001$ different from 0 ). Error bars in this and the following figures show s.e.m. For detailed captions, see Supplementary table 1: Experiment summary and SM3: Statistics. 

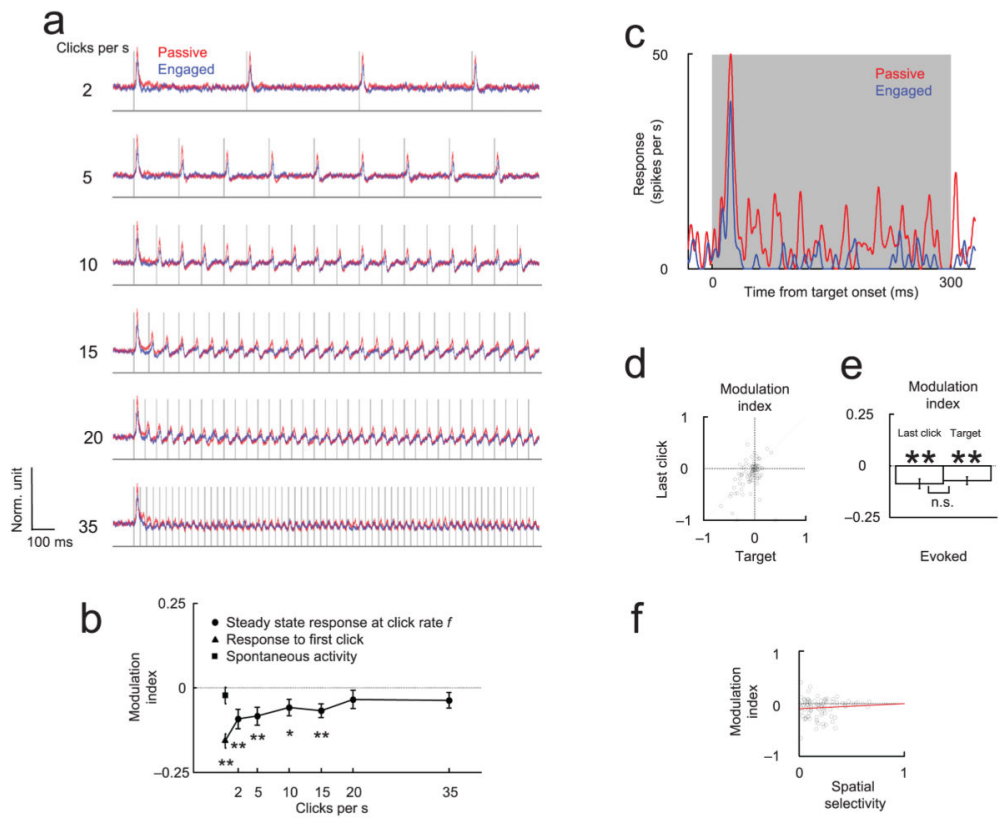

Figure 2. Decision-relevant target is suppressed in engaged condition

(a) Responses evoked by clicks (i.e. task-irrelevant distractors) are attenuated at higher repetition rates in both the engaged (blue) and passive ( $r e d$ ) conditions (Task 1). The traces show the average normalized PSTH of cortical multiunit responses ( $N=60$ sites) to six different repetition rates. Line thickness is proportional to s.e.m. (b) Task-dependent suppression (modulation index) of the click-evoked responses decreases at higher stimulation rates. The square and triangle symbols indicate the modulation index for spontaneous firing and the first stimulus, respectively. (c) Example of multiunit cortical response to contralateral task-relevant stimulus. Responses to ipsilateral stimuli were generally weak and were not analyzed. (d-e) The modulation of the target stimulus is correlated with the modulation of the preceding (task-irrelevant) stimulus (d) and has a comparable magnitude (e). (f) Spatial selectivity and task-engaged suppression are statistically uncorrelated (regression line in red in this and following figures), indicating that selective responses were not preferentially enhanced during the task. Spatial selectivity was calculated between the left and right target stimulus during the passive condition. We quantified the spatial selectivity using the absolute selectivity, defined as $2 *$ abs ((area under the ROC curve)-0.5) ${ }^{50}$. This quantity is zero if the response was not selective between the left and the right target stimulus and 1 if the response was perfectly selective. (See also Fig. $\mathrm{S} 2$ : Single unit responses to task relevant and task irrelevant stimuli are equally suppressed during the task). 


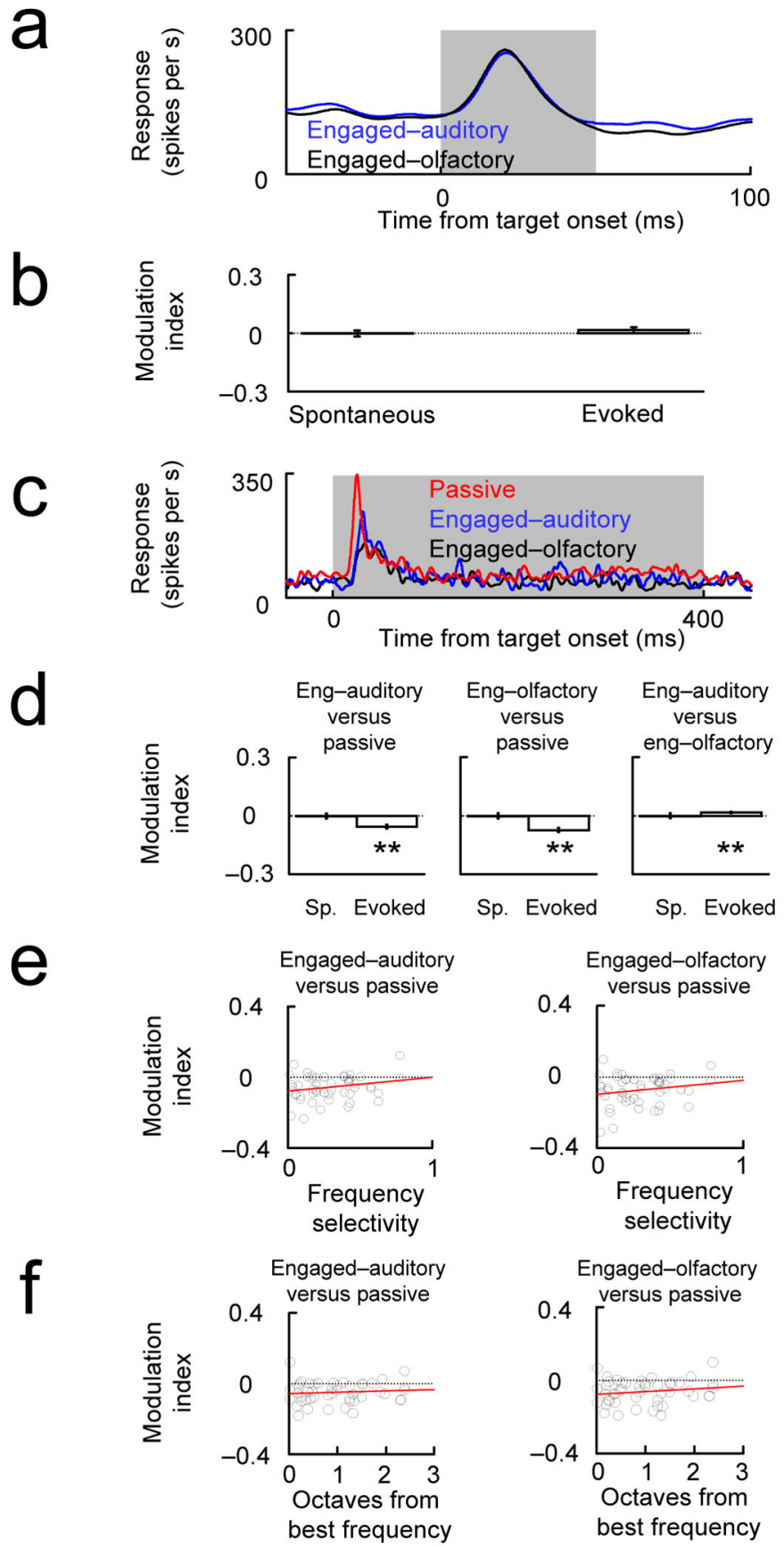

Figure 3. (a, b) Evoked auditory responses are not suppressed during an auditory task relative to an olfactory task

Animals performed interleaved blocks ( 50-70 trials) of an auditory task (engagedauditory) and an olfactory task (engaged-olfactory). In some experiments ( $c-f$, Task $\mathbf{3}$ ) a passive block was also tested. Auditory stimuli (a high and a low tone) were identical in the two (or three) blocks. (a) Example tone-evoked multiunit response in auditory cortex shows no difference between the auditory and olfactory blocks (Task 2). (b) The modulation index $\left(\left(\right.\right.$ Activity $_{\text {auditory block }}-$ Activity $\left._{\text {olfactory block }}\right) /\left(\right.$ Activity $_{\text {auditory block }}+$ Activity $\left.\left._{\text {olfactory block }}\right)\right)$ 
showed no difference between the engaged-auditory and engaged-olfactory conditions (compare engaged vs. passive, Fig. 1; see also SM6: Single unit responses during the intermodal auditory-olfactory task and SM3: Statistics for more detailed figure captions). (c-d) Example PSTH and population data showing that engaged-auditory and engaged-olfactory responses were suppressed relative to the passive condition. (e) There was no significant correlation between frequency selectivity and the (engaged vs. passive) modulation index. Frequency selectivity was calculated during the passive condition between the two pure tone stimulus used as targets We quantified the frequency selectivity using the absolute selectivity, defined as $2 *$ abs $((\text { area under the ROC curve })-0.5)^{50}$. This quantity is zero if the response was not selective between the high and the low tone and 1 if the response was perfectly selective. (f) We measured frequency tuning curves at each site (see Fig. S3: Measurement of cortical tuning curves) and found no correlation between the modulation during the task for a particular tone and the distance to the best frequency in octaves. 

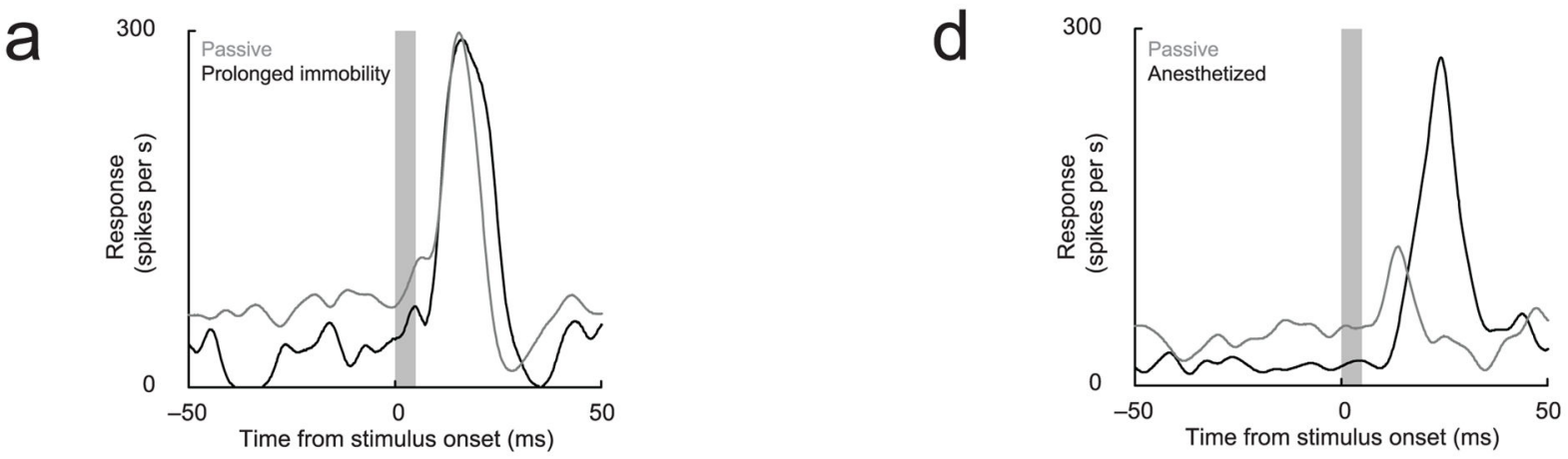

b
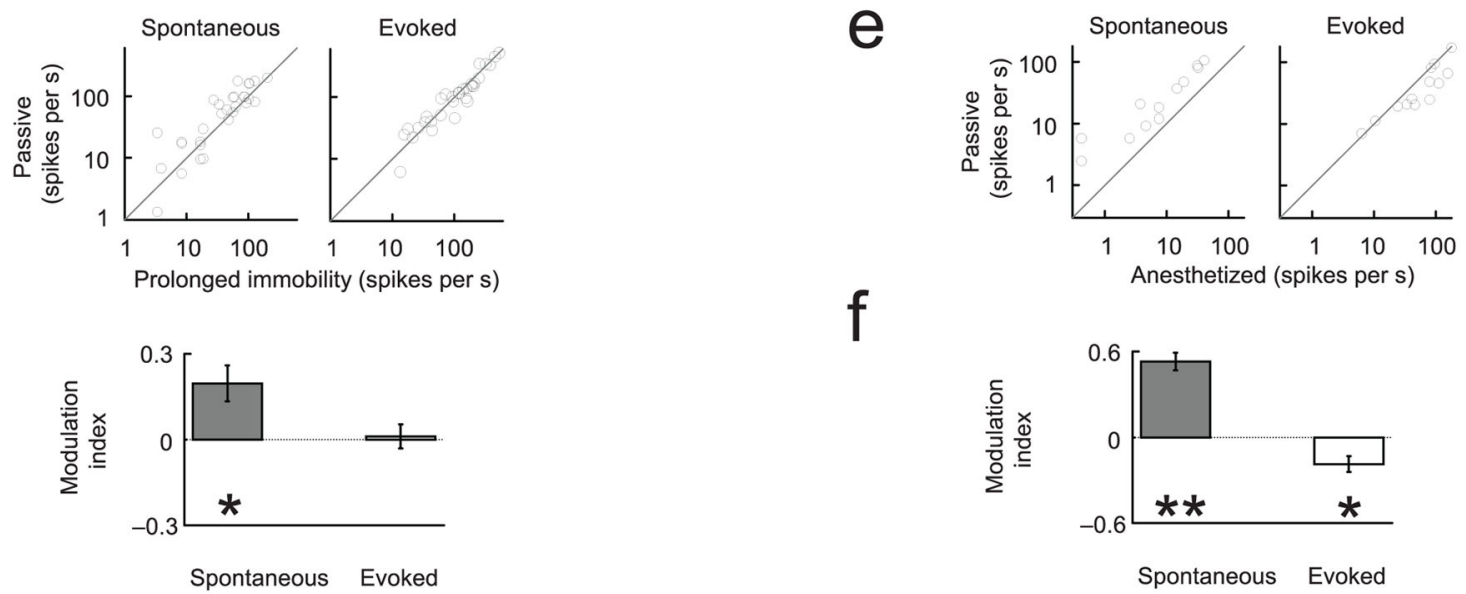

Figure 4. Changes in arousal and anesthesia have distinct neural signatures (a-c) Spontaneous but not evoked multiunit responses were suppressed relative to the passive condition during prolonged immobility, possibly associated with sleep. The

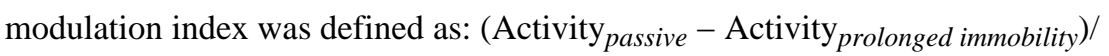

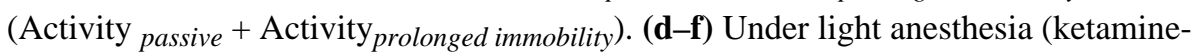
medetomidine), spontaneous firing rates were also suppressed, and evoked responses were enhanced, relative to the passive condition. The modulation index was defined as: $\left(\right.$ Activity $_{\text {passive }}-$ Activity $\left._{\text {anesthetized }}\right) /\left(\right.$ Activity $_{\text {passive }}+$ Activity $\left._{\text {anesthetized }}\right)$. 


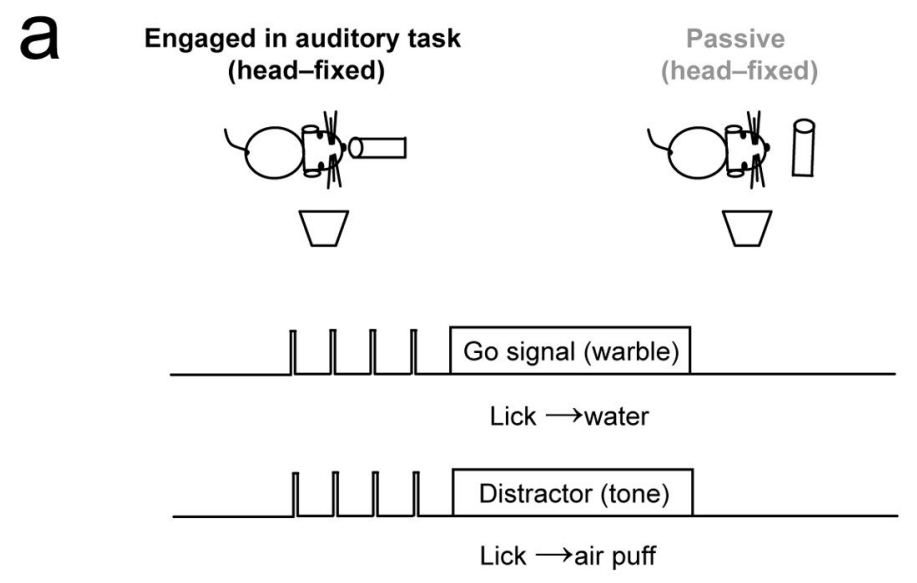

b
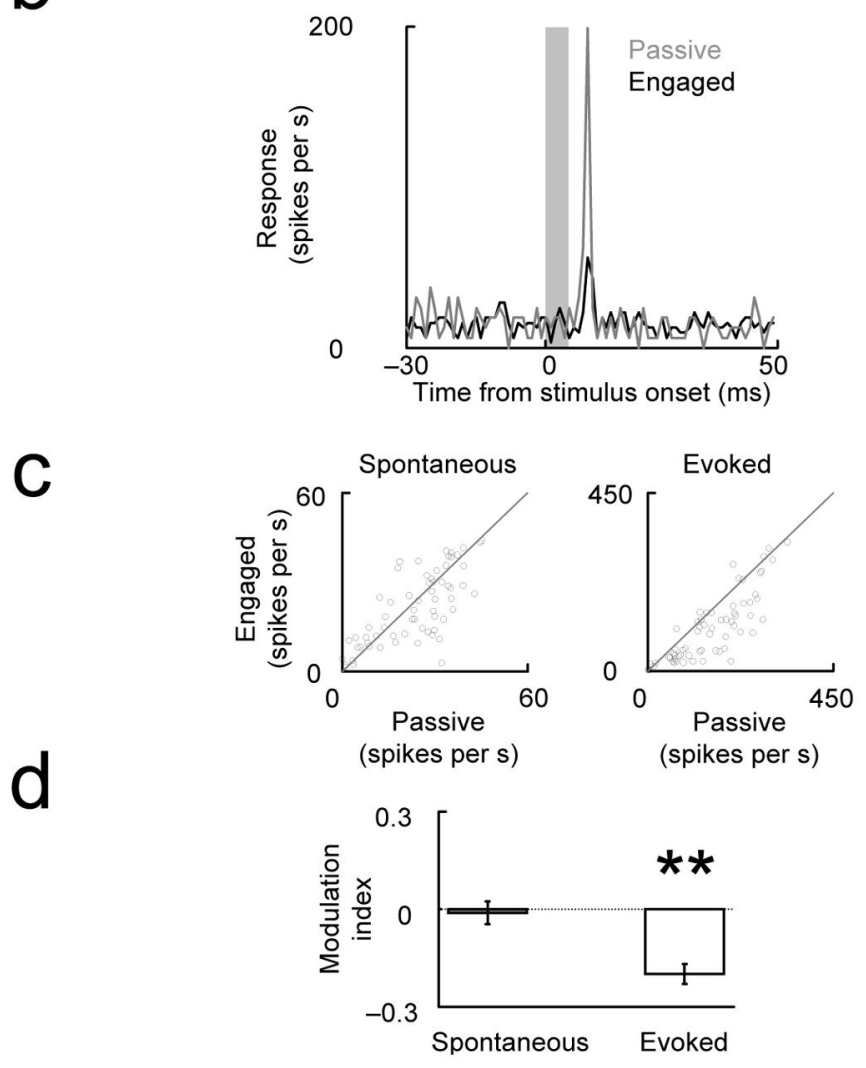

Figure 5. Suppression of evoked responses is not caused by self-triggering of stimulus (a) Head-fixed animals performed a Go/No-Go auditory discrimination task (Task 4). The stimuli started randomly and were not triggered by the subject. Multiunit responses in the engaged-auditory condition were compared with those to the same stimuli presented when the water delivery system was withdrawn (passive). (b-d) Task-engaged suppression of the evoked response was observed, comparable to that seen in Fig 1. (Format parallel to that in Fig. 1. See also Fig. S5: Example and population data showing suppression of sound evoked LFPs in the head-fixed behavior for LFP analysis.) 

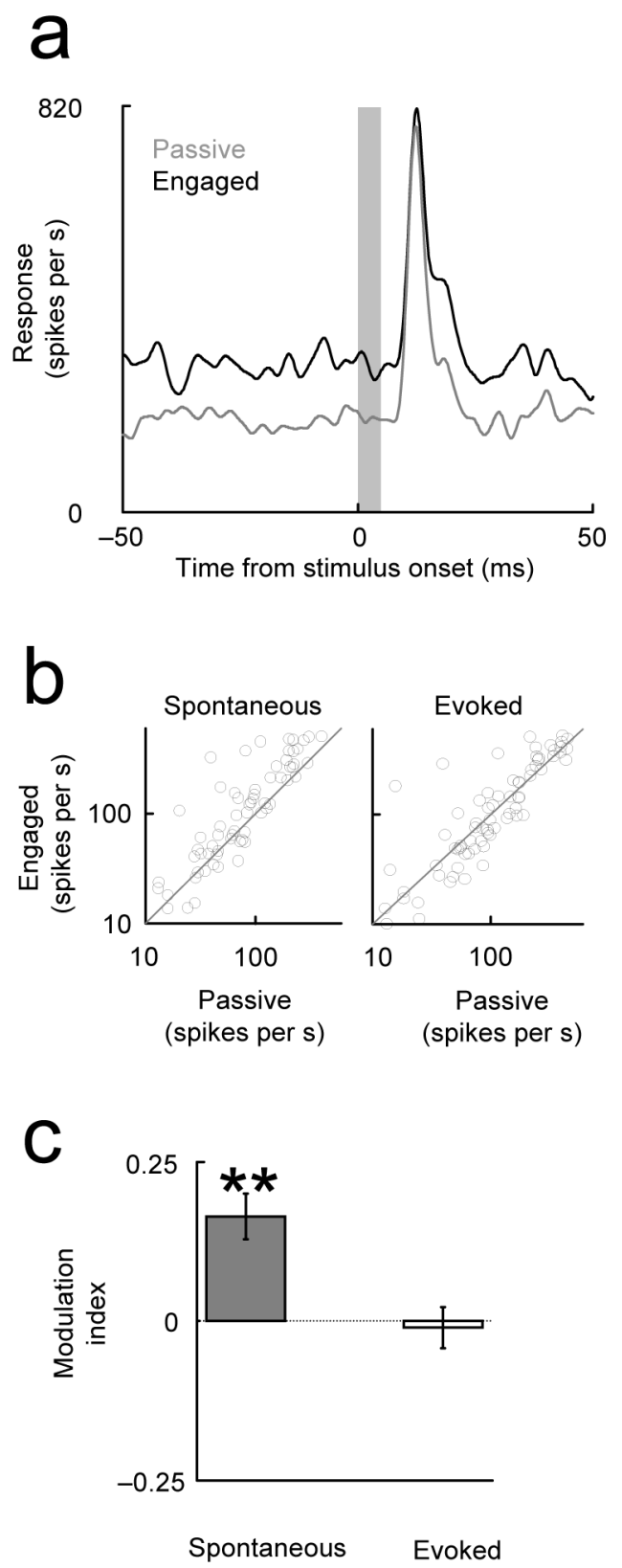

Figure 6. Neural correlate of engagement differs in auditory thalamus (a) Thalamic spontaneous responses were elevated in the engaged condition (Task 1) but evoked responses were unchanged. (top) Example multiunit thalamic peri-stimulus time histogram (PSTH). (bottom) Population analysis. (Format parallel to that in Fig. 1; see legend for details). 
Table 1

\begin{tabular}{|c|c|c|}
\hline Auditory Cortex & Evoked activity & Spontaneous activity \\
\hline passive vs. engaged auditory & $\uparrow$ & $=$ \\
\hline passive vs. engaged olfactory & $\uparrow$ & $=$ \\
\hline passive vs. immobile & $=$ & $\uparrow$ \\
\hline passive vs. anesthetized & $\downarrow$ & $\uparrow$ \\
\hline engaged auditory vs. engaged olfactory & $\uparrow$ & $=$ \\
\hline Auditory Thalamus & & \\
\hline passive vs. engaged & $=$ & $\downarrow$ \\
\hline
\end{tabular}

